\title{
Spectrum of hidden-charm, open-strange exotics in the dynamical diquark model
}

\author{
Jesse F. Giron $\odot,{ }^{*}$ Richard F. Lebed $\odot,^{\dagger}$ and Steven R. Martinez $\oplus^{\ddagger}$ \\ Department of Physics, Arizona State University, Tempe, Arizona 85287, USA
}

(Received 15 June 2021; accepted 4 August 2021; published 2 September 2021)

\begin{abstract}
The observation by BESIII and LHCb of states with hidden charm and open strangeness $(c \bar{c} q \bar{s})$ presents new opportunities for the development of a global model of heavy-quark exotics. Here we extend the dynamical diquark model to encompass such states, using the same values of Hamiltonian parameters previously obtained from the nonstrange and hidden-strange sectors. The large mass splitting between $Z_{c s}(4000)$ and $Z_{c s}(4220)$ suggests substantial $\mathrm{SU}(3)_{\text {flavor }}$ mixing between all $J^{P}=1^{+}$states, while their average mass compared to that of other sectors offers a direct probe of flavor octet-singlet mixing among exotics. We also explore the inclusion of $\eta$-like exchanges within the states, and find their effects to be quite limited. In addition, using the same diquark-mass parameters, we find $P_{c}(4312)$ and $P_{c s}(4459)$ to fit well as corresponding nonstrange and open-strange pentaquarks.
\end{abstract}

DOI: $10.1103 /$ PhysRevD.104.054001

\section{INTRODUCTION}

The spectrum of known heavy-quark exotic hadrons continues to expand frequently, with about 50 candidates observed to date. Almost all have a valence light-flavor content consisting of only $q \equiv u$ and $d$ quarks, but very recently some states with open strangeness have been detected in both the open-charm [1,2] $(\bar{c} d \bar{s} u)$ and hiddencharm sectors. In the latter, both a pentaquark [3] ( $c \bar{c} u d s$ ) state and tetraquark [4,5] $(c \bar{c} u \bar{s})$ states have been observed. ${ }^{1}$

Multiple reviews summarizing both experimental and theoretical advances in this field have appeared in recent years [6-16]. Several competing theoretical frameworks (dihadron molecular states, bound states of diquarks, threshold enhancements, etc.) have been developed for years, but no single scheme has yet emerged as a dominant paradigm to explain all the new states, analogous to the way that quark-potential models successfully elucidate the conventional $c \bar{c}$ and $b \bar{b}$ sectors [17].

Since a number of the observed exotics decay to final states like $J / \psi \phi$ or $D_{s J}^{(*)} \bar{D}_{s J}^{(*)}$, they possess a presumptive $c \bar{c} s \bar{s}$ valence content. The advent of $\bar{c} c \bar{s} q$ states thus

\footnotetext{
*jfgiron@asu.edu

Richard.Lebed@asu.edu

*srmart16@asu.edu

${ }^{1}$ Charge-conjugate states are implied as included throughout.
}

Published by the American Physical Society under the terms of the Creative Commons Attribution 4.0 International license. Further distribution of this work must maintain attribution to the author(s) and the published article's title, journal citation, and DOI. Funded by SCOAP ${ }^{3}$. introduces an intermediate case between $c \bar{c} q \bar{q}^{\prime}$ and $c \bar{c} s \bar{s}$ cases, and therefore not only provides an opportunity to examine whether a particular theoretical picture can successfully incorporate data from all of these flavor sectors, but also examines the manifestation of lightquark $\mathrm{SU}(3)_{\text {flavor }}$ for the first time outside of conventional mesons and baryons.

The new data in the hidden-charm, open-strange sector itself is quite interesting. BESIII observes a structure [4] in the $K^{+}$recoil spectrum of $e^{+} e^{-} \rightarrow K^{+}\left(D_{s}^{-} D^{* 0}+D_{s}^{*-} D^{0}\right)$ near the two-charmed-meson thresholds (about 3975 and $3977 \mathrm{MeV}$, respectively). For this $Z_{c s}(3985)$ state, they obtain

$$
\begin{aligned}
m_{Z_{c s}^{-}} & =3982.5_{-2.6}^{+1.8} \pm 2.1 \mathrm{MeV}, \\
\Gamma_{Z_{c s}^{-}} & =12.8_{-4.4}^{+5.3} \pm 3.0 \mathrm{MeV},
\end{aligned}
$$

and $J^{P}=1^{+}$. Meanwhile, LHCb reports two states [5] decaying to $J / \psi K^{+}$,

$$
\begin{aligned}
m_{Z_{c s}^{+}(4000)} & =4003 \pm 6_{-14}^{+4} \mathrm{MeV}, \\
\Gamma_{Z_{c s}^{+}(4000)} & =131 \pm 15 \pm 26 \mathrm{MeV}, \\
m_{Z_{c s}^{+}(4220)} & =4216 \pm 24_{-30}^{+43} \mathrm{MeV}, \\
\Gamma_{Z_{c s}^{+}(4220)} & =233 \pm 52_{-73}^{+97} \mathrm{MeV},
\end{aligned}
$$

with $Z_{c s}(4000)$ carrying $J^{P}=1^{+}$and $Z_{c s}(4220)$ favored to carry $J^{P}=1^{+}$. The masses of $Z_{c s}(3985)$ and $Z_{c s}(4000)$ are compatible with them being the same state, but their measured widths are wildly different. For the purposes of this paper, we assume that only a single light $Z_{c s}$ state 
exists near $4.0 \mathrm{GeV}$, the discrepancy in width measurements perhaps arising from effects caused by interactions with the nearby charmed-meson thresholds. ${ }^{2}$ LHCb also observes a baryonic structure [3] $P_{c s}(4459)$ decaying to $J / \psi \Lambda$ :

$$
\begin{aligned}
m_{P_{c s}(4459)} & =4458.8 \pm 2.9_{-1.1}^{+4.7} \mathrm{MeV}, \\
\Gamma_{P_{c s}(4459)} & =17.3 \pm 6.5_{-5.7}^{+8.0} \mathrm{MeV},
\end{aligned}
$$

although its $J^{P}$ value is not yet determined.

Quite an extensive body of theoretical work on the hidden-charm, open-strange hadrons exists. For example, in the meson sector whose study forms the bulk of this work, several papers [19-24] predate the experimental observations, while multiple studies followed the announcement of the BESIII result but preceded the appearance of the LHCb paper [25-47], and yet others appeared subsequent to the LHCb results [18,48-55]. As one may imagine, this body of work encompasses multiple approaches, including molecular and diquark models, chiral-quark models, and QCD sum rules, among others.

The present work uses the dynamical diquark model, initially introduced in Ref. [56] as a theoretical picture to explain how relatively compact color-triplet diquark quasiparticle pairs can form spatially extended tetraquark states, and extended in Ref. [57] to describe pentaquarks as color-triplet diquark-triquark quasiparticle bound states. The picture is developed in Ref. [58] into a predictive model by noting that the static interaction potential between the heavy color-triplet quasiparticles is the same one as appearing in lattice simulations of heavy quarkonium and its hybrids. The multiplet band structure for $c \bar{c} q \bar{q}^{\prime}$ and $c \bar{c} q q q$ states is studied numerically in Ref. [59]; the fine structure of the ground-state ( $S$-wave) $c \bar{c} q \bar{q}^{\prime}$ multiplet is examined numerically in Ref. [60] and that of the $P$-wave multiplet appears in Ref. [61]. An analogous study of the $b \bar{b} q \bar{q}^{\prime}$ and $c \bar{c} s \bar{s}$ systems is presented in Ref. [62], and the $c \bar{c} c \bar{c}$ states are investigated in Ref. [63]. Radiative transitions between exotic states are computed in Ref. [64].

Our analysis of $c \bar{c} q \bar{s}$ exotics here directly interpolates between the analysis of $c \bar{c} q \bar{q}^{\prime}$ in Ref. [60] and $c \bar{c} s \bar{s}$ in Ref. [62], and uses the same numerical inputs. However, we find that a careful treatment of the $\mathrm{SU}(3)_{\text {flavor }}$ structure introduces one new parameter, related to octet-singlet mixing. In addition, we allow for the possibility of an $\eta$-like exchange between the diquarks analogous to the $\pi$-like exchange already present in the original model [60] but show that its effects are quite limited by constraints from the phenomenology of the $c \bar{c} q \bar{q}^{\prime}$ sector. We find that

\footnotetext{
${ }^{2}$ This opinion is not universal. For example, Ref. [18] treats $Z_{c s}(3985)$ and $Z_{c s}(4000)$ as separate states belonging to distinct $\mathrm{SU}(3)_{\text {flavor }}$ multiplets.
}

the known phenomenology of the open-strange sector does indeed follow from that of the other sectors, despite superficially appearing quite different. We also carry out an analogous exercise for nonstrange and open-strange hidden-charm pentaquark states $\left[P_{c s}(4459)\right.$ currently being the only known example of the latter], and obtain remarkably satisfactory results.

This paper is organized as follows. In Sec. II we define the multiplets of states in terms of eigenstates of both good diquark spin and good heavy-quark/light-quark spins. Section III presents the Hamiltonian for the $c \bar{c} q \bar{q}^{\prime}$ and $c \bar{c} s \bar{s}$ sectors, now including a possible term from $\eta$-like exchanges, and computes all relevant matrix elements. Section IV performs the same analysis for the $c \bar{c} q \bar{s}$ sector, and discusses possible mixing between multiplets whose nonstrange members carry opposite $C$ parity. In Sec. V we discuss the effects of octet-singlet mixing on the analysis and present numerical results, and in Sec. VI we summarize and conclude.

\section{STATES OF THE MODEL}

A cataloguing of the $Q \bar{Q} q \bar{q}^{\prime}$ or $Q \bar{Q} q q_{1} q_{2}$ states in the dynamical diquark model, where $q, q^{\prime}, q_{i} \in\{u, d\}$, first appears in Ref. [58]. The same notation, with small modifications, is applied to $c \bar{c} s \bar{s}$ in Ref. [62] and to $c \bar{c} c \bar{c}$ in Ref. [63]. All confirmed exotic candidates to date have successfully been accommodated within the lowest $\left(\Sigma_{g}^{+}\right)$Born-Oppenheimer potential of the gluon field connecting the heavy diquark $[\delta \equiv(Q q)]$-antidiquark $\left[\bar{\delta} \equiv\left(\bar{Q} \bar{q}^{\prime}\right)\right]$ or diquark-triquark $\left[\bar{\theta} \equiv\left(\bar{Q}\left(q_{1} q_{2}\right)\right)\right]$ quasiparticles. In all cases, $\delta, \bar{\delta}, \bar{\theta}$ are assumed to transform as color triplets (or antitriplets) and each quasiparticle contains no internal orbital angular momentum.

In the case of $Q \bar{Q} q \bar{q}^{\prime}$, the classification scheme then begins with six possible core states in which the quasiparticle pair lie in a relative $S$ wave. Indicating the total spin $s$ of a diquark $\delta, \bar{\delta}$ by $s_{\delta}, s_{\bar{\delta}}$ and using a subscript on the full state to indicate its total spin, one obtains the spectrum

$$
\begin{aligned}
J^{P C}=0^{++}: X_{0} & \equiv\left|0_{\delta}, 0_{\bar{\delta}}\right\rangle_{0}, \quad X_{0}^{\prime} \equiv\left|1_{\delta}, 1_{\bar{\delta}}\right\rangle_{0}, \\
J^{P C}=1^{++}: X_{1} & \equiv \frac{1}{\sqrt{2}}\left(\left|1_{\delta}, 0_{\bar{\delta}}\right\rangle_{1}+\left|0_{\delta}, 1_{\bar{\delta}}\right\rangle_{1}\right), \\
J^{P C}=1^{+-}: Z & \equiv \frac{1}{\sqrt{2}}\left(\left|1_{\delta}, 0_{\bar{\delta}}\right\rangle_{1}-\left|0_{\delta}, 1_{\bar{\delta}}\right\rangle_{1}\right), \\
Z^{\prime} & \equiv\left|1_{\delta}, 1_{\bar{\delta}}\right\rangle_{1}, \\
J^{P C}=2^{++}: X_{2} & \equiv\left|1_{\delta}, 1_{\bar{\delta}}\right\rangle_{2} .
\end{aligned}
$$

Since 4 quark angular momenta are being combined, one may transform these states into other convenient bases by means of $9 j$ angular momentum recoupling coefficients. In particular, in the basis of good total heavy-quark $(Q \bar{Q})$ and light-quark $\left(q \bar{q}^{\prime}\right)$ spin, the transformation reads 


$$
\begin{gathered}
\left\langle\left(s_{q} s_{\bar{q}}\right) s_{q \bar{q}^{\prime}},\left(s_{Q} s_{\bar{Q}}\right) s_{Q \bar{Q}}, S \mid\left(s_{q} s_{Q}\right) s_{\delta},\left(s_{\bar{q}} s_{\bar{Q}}\right) s_{\bar{\delta}}, S\right\rangle \\
=\left(\left[s_{q \bar{q}^{\prime}}\right]\left[s_{Q \bar{Q}}\right]\left[s_{\delta}\right]\left[s_{\bar{\delta}}\right]\right)^{1 / 2}\left\{\begin{array}{ccc}
s_{q} & s_{\bar{q}} & s_{q \bar{q}^{\prime}} \\
s_{Q} & s_{\bar{Q}} & s_{Q \bar{Q}} \\
s_{\delta} & s_{\bar{\delta}} & S
\end{array}\right\},
\end{gathered}
$$

with $[s] \equiv 2 s+1$ signifying the multiplicity of a spin- $s$ state. Using Eqs. (4) and (5), one then obtains

$$
\begin{gathered}
J^{P C}=0^{++}: X_{0}=\frac{1}{2}\left|0_{q \bar{q}^{\prime}}, 0_{Q \bar{Q}}\right\rangle_{0}+\frac{\sqrt{3}}{2}\left|1_{q \bar{q}^{\prime}}, 1_{Q \bar{Q}}\right\rangle_{0}, \\
X_{0}^{\prime}=\frac{\sqrt{3}}{2}\left|0_{q \bar{q}^{\prime}}, 0_{Q \bar{Q}}\right\rangle_{0}-\frac{1}{2}\left|1_{q \bar{q}^{\prime}}, 1_{Q \bar{Q}}\right\rangle_{0}, \\
J^{P C}=1^{++}: X_{1}=\left|1_{q \bar{q}^{\prime}}, 1_{Q \bar{Q}}\right\rangle_{1}, \\
J^{P C}=1^{+-}: Z=\frac{1}{\sqrt{2}}\left(\left|1_{q \bar{q}^{\prime}}, 0_{Q \bar{Q}}\right\rangle_{1}-\left|0_{q \bar{q}^{\prime}}, 1_{Q \bar{Q}}\right\rangle_{1}\right), \\
Z^{\prime}=\frac{1}{\sqrt{2}}\left(\left|1_{q \bar{q}^{\prime}}, 0_{Q \bar{Q}}\right\rangle_{1}+\left|0_{q \bar{q}^{\prime}}, 1_{Q \bar{Q}}\right\rangle_{1}\right), \\
J^{P C}=2^{++}: X_{2}=\left|1_{q \bar{q}^{\prime}}, 1_{Q \bar{Q}}\right\rangle_{2} .
\end{gathered}
$$

In this work it is especially convenient to employ a basis of states carrying a unique value of $s_{Q \bar{Q}}$ and of $s_{q \bar{q}^{\prime}}$. These states are $X_{1}$ and $X_{2}$ [as seen in Eq. (6)], and

$$
\begin{aligned}
& \tilde{X}_{0} \equiv\left|0_{q \bar{q}^{\prime}}, 0_{Q \bar{Q}}\right\rangle_{0}=+\frac{1}{2} X_{0}+\frac{\sqrt{3}}{2} X_{0}^{\prime}, \\
& \tilde{X}_{0}^{\prime} \equiv\left|1_{q \bar{q}^{\prime}}, 1_{Q \bar{Q}}\right\rangle_{0}=+\frac{\sqrt{3}}{2} X_{0}-\frac{1}{2} X_{0}^{\prime}, \\
& \tilde{Z} \equiv\left|1_{q \bar{q}^{\prime}}, 0_{Q \bar{Q}}\right\rangle_{1}=\frac{1}{\sqrt{2}}\left(Z^{\prime}+Z\right), \\
& \tilde{Z}^{\prime} \equiv\left|0_{q \bar{q}^{\prime}}, 1_{Q \bar{Q}}\right\rangle_{1}=\frac{1}{\sqrt{2}}\left(Z^{\prime}-Z\right) .
\end{aligned}
$$

Including $(u, d)$ the light-quark flavor produces 12 states: six each with $I=0$ and $I=1$, and spin structures in the form of Eqs. (4), (6), or (7). The basis of Eq. (7) in particular is ideal for discussing $\mathrm{SU}(3)_{\text {flavor }}$ multiplets: a state component like $1_{u \bar{s}}$ is a pure flavor octet that transforms under spin and flavor analogously to $K^{*+}$ (although in a diquark model it comprises a mixture of color-singlet and color-octet components). The full SU(3) flavor structure of the multiplet $\Sigma_{g}^{+}(1 S)$ thus consists of six octets and six singlets. A study of the possible mixing of states with the same $J^{P}$ between different $\mathrm{SU}(3)_{\text {flavor }}$ octets, or of octetsinglet mixing, form two principal theory innovations of this work.

The $Q \bar{Q} q \bar{q}^{\prime}$ states in the multiplet $\Sigma_{g}^{+}(1 S)$ are sufficient to accommodate all particles considered in this work. However, we note that Ref. [58] also provides a classification of orbitally excited states [the multiplets $\Sigma_{g}^{+}(n P)$ appearing in Ref. [61] ], as well as states in excited-glue Born-Oppenheimer potentials such as $\Pi_{u}^{+}$(which are exotic analogues to hybrid mesons), and pentaquark states $Q \bar{Q} q q_{1} q_{2}$.

\section{REVIEW OF $c \bar{c} q \bar{q}^{\prime}$ AND $c \bar{c} s \bar{s}$ SECTOR}

\section{A. $c \bar{c} q \bar{q}^{\prime}$ sector}

For hidden heavy-flavor exotics containing only $u$ and/or $d$ light valence quarks, we write the following Hamiltonian:

$$
\begin{aligned}
H= & M_{0}+\Delta H_{\kappa_{q Q}}+\Delta H_{V_{0}}+\Delta H_{V_{8}}, \\
= & M_{0}+2 \kappa_{q Q}\left(\mathbf{s}_{q} \cdot \mathbf{s}_{Q}+\mathbf{s}_{\bar{q}} \cdot \mathbf{s}_{\bar{Q}}\right)+V_{0}\left(\boldsymbol{\tau}_{q} \cdot \boldsymbol{\tau}_{\bar{q}}\right)\left(\boldsymbol{\sigma}_{q} \cdot \boldsymbol{\sigma}_{\bar{q}}\right) \\
& +V_{8}\left(\lambda_{a}^{8} \boldsymbol{\sigma}_{q}\right) \cdot\left(\lambda_{a}^{8} \boldsymbol{\sigma}_{\bar{q}}\right) .
\end{aligned}
$$

Here, $M_{0}$ is the common $\Sigma_{g}^{+}(1 S)$ multiplet mass, which depends only upon the chosen diquark $(\delta, \bar{\delta})$ masses and a central potential $V(r)$ computed numerically on the lattice from pure glue configurations that connect $\mathbf{3}$ and $\overline{\mathbf{3}}$ sources, as employed in Ref. [59]. The second term represents the spin-spin interaction within diquarks, assumed to couple only $q \leftrightarrow Q$ and $\bar{q}^{\prime} \leftrightarrow \bar{Q}$, and $\kappa_{q Q}$ indicates the strength of this interaction. The prime (flavor) index on the light antiquark has been suppressed throughout Eq. (8), since for the moment we consider $q, q^{\prime}$ to be either a light-quark or strange-quark pair, so that the same value of $\kappa_{q Q}$ appears for both spin-spin terms. An isospin-spin-dependent interaction of strength $V_{0}$ between the light-quark spins, which is modeled on the pion-nucleon coupling and was first introduced in Ref. [60], comprises the third term. These three terms form the full set included in the analysis of Ref. [60]. The final term is new to this work; it is modeled on an $\eta$-nucleon coupling and evaluates in the relevant flavor sectors to

$\Delta M_{V_{8}}=\frac{1}{3} V_{8}\left[2 s_{q \bar{q}^{\prime}}\left(s_{q \bar{q}^{\prime}}+1\right)-3\right] \times\left\{\begin{array}{ll}1, & q, q^{\prime} \in\{u, d\} \\ 4, & q, q^{\prime}=s, s\end{array}\right.$.

To compute the mass expressions arising from Eq. (8), let us first abbreviate

$$
V_{-} \equiv V_{0}-\frac{1}{9} V_{8}, \quad V_{+} \equiv V_{0}+\frac{1}{3} V_{8}
$$

Then the Hamiltonian matrix elements for the mixed $Q \bar{Q} q \bar{q}^{\prime}$ states of the $\Sigma_{g}^{+}(1 S)$ multiplet, their components arranged in the order $s_{Q \bar{Q}}=0,1$, read 


$$
\begin{aligned}
& \tilde{M}_{0^{++}}^{I=0}=M_{0}\left(\begin{array}{ll}
1 & 0 \\
0 & 1
\end{array}\right)-\kappa_{q Q}\left(\begin{array}{cc}
0 & \sqrt{3} \\
\sqrt{3} & 2
\end{array}\right)-3 V_{-}\left(\begin{array}{cc}
-3 & 0 \\
0 & 1
\end{array}\right), \\
& \tilde{M}_{0^{++}}^{I=1}=M_{0}\left(\begin{array}{ll}
1 & 0 \\
0 & 1
\end{array}\right)-\kappa_{q Q}\left(\begin{array}{cc}
0 & \sqrt{3} \\
\sqrt{3} & 2
\end{array}\right)+V_{+}\left(\begin{array}{cc}
-3 & 0 \\
0 & 1
\end{array}\right), \\
& \tilde{M}_{1^{++}}^{I=0}=M_{0}\left(\begin{array}{ll}
1 & 0 \\
0 & 1
\end{array}\right)+\kappa_{q Q}\left(\begin{array}{ll}
0 & 1 \\
1 & 0
\end{array}\right)-3 V_{-}\left(\begin{array}{cc}
1 & 0 \\
0 & -3
\end{array}\right), \\
& \tilde{M}_{1^{++}}^{I=1}=M_{0}\left(\begin{array}{ll}
1 & 0 \\
0 & 1
\end{array}\right)+\kappa_{q Q}\left(\begin{array}{ll}
0 & 1 \\
1 & 0
\end{array}\right)+V_{+}\left(\begin{array}{cc}
1 & 0 \\
0 & -3
\end{array}\right) .
\end{aligned}
$$

Diagonalizing the expressions of Eq. (11) in order of increasing mass and appending the corresponding (already diagonal) expressions for the remaining $Q \bar{Q} q \bar{q}^{\prime}$ states of the $\Sigma_{g}^{+}(1 S)$ multiplet, one obtains

$M_{0^{++}}^{I=0}=\left(M_{0}-\kappa_{q Q}+3 V_{-}\right)\left(\begin{array}{ll}1 & 0 \\ 0 & 1\end{array}\right)+2 \tilde{V}_{1}^{Q \bar{Q} q \bar{q}}\left(\begin{array}{cc}-1 & 0 \\ 0 & 1\end{array}\right)$,

$M_{0^{++}}^{I=1}=\left(M_{0}-\kappa_{q Q}-V_{+}\right)\left(\begin{array}{ll}1 & 0 \\ 0 & 1\end{array}\right)+2 \tilde{V}_{2}^{Q \bar{Q} q \bar{q}}\left(\begin{array}{cc}-1 & 0 \\ 0 & 1\end{array}\right)$,

$M_{1^{+-}}^{I=0}=\left(M_{0}+3 V_{-}\right)\left(\begin{array}{ll}1 & 0 \\ 0 & 1\end{array}\right)+\tilde{V}_{3} Q \bar{Q} q \bar{q}\left(\begin{array}{cc}-1 & 0 \\ 0 & 1\end{array}\right)$,

$M_{1^{+-}}^{I=1}=\left(M_{0}-V_{+}\right)\left(\begin{array}{ll}1 & 0 \\ 0 & 1\end{array}\right)+\tilde{V}_{4}^{Q \bar{Q} q \bar{q}}\left(\begin{array}{cc}-1 & 0 \\ 0 & 1\end{array}\right)$,

$M_{1^{++}}^{I=0}=M_{0}-\kappa_{q Q}-3 V_{-}$,

$M_{1^{++}}^{I=1}=M_{0}-\kappa_{q Q}+V_{+}$,

$M_{2^{++}}^{I=0}=M_{0}+\kappa_{q Q}-3 V_{-}$,

$M_{2^{++}}^{I=1}=M_{0}+\kappa_{q Q}+V_{+}$,

using the abbreviations

$$
\begin{aligned}
& \tilde{V}_{1}^{Q \bar{Q} q \bar{q}} \equiv \sqrt{\kappa_{q Q}{ }^{2}+3 \kappa_{q Q} V_{-}+9 V_{-}^{2}}, \\
& \tilde{V}_{2}^{Q \bar{Q} q \bar{q}} \equiv \sqrt{\kappa_{q Q}{ }^{2}-\kappa_{q Q} V_{+}+V_{+}^{2}}, \\
& \tilde{V}_{3}^{Q \bar{Q} q \bar{q}} \equiv \sqrt{\kappa_{q Q}{ }^{2}+36 V_{-}^{2}}, \\
& \tilde{V}_{4}^{Q \bar{Q} q \bar{q}} \equiv \sqrt{\kappa_{q Q}{ }^{2}+4 V_{+}^{2}} .
\end{aligned}
$$

\section{B. $c \bar{c} s \bar{s}$ sector}

The Hamiltonian relevant to the $c \bar{c} s \bar{s}$ sector is identical to the one in Eq. (8), omitting the isospin-dependent $V_{0}$ term and performing some $q \rightarrow s$ relabeling,

$$
\begin{aligned}
H & =M_{0}+\Delta H_{\kappa_{s}}+\Delta H_{V_{0}}+\Delta H_{V_{8}}, \\
& =M_{0}+2 \kappa_{s Q}\left(\mathbf{s}_{s} \cdot \mathbf{s}_{Q}+\mathbf{s}_{\bar{s}} \cdot \mathbf{s}_{\bar{Q}}\right)+V_{8}\left(\lambda_{a}^{8} \boldsymbol{\sigma}_{s}\right) \cdot\left(\lambda_{a}^{8} \boldsymbol{\sigma}_{\bar{s}}\right) .
\end{aligned}
$$

Equivalently, this expression generalizes the Hamiltonian used in the analysis of Ref. [62] by the inclusion of the $V_{8}$ term. Note that the value of $M_{0}=M_{0}^{Q \bar{Q}} s \bar{s}$ here differs from $M_{0}=M_{0}^{Q \bar{Q} q \bar{q}}$ appearing in Eq. (8). The mass eigenvalues for the six isosinglet $c \bar{c} s \bar{s}$ states evaluate to

$$
\begin{aligned}
M_{0^{++}} & =\left(M_{0}-\kappa_{s Q}-\frac{4}{3} V_{8}\right)\left(\begin{array}{ll}
1 & 0 \\
0 & 1
\end{array}\right)+2 \tilde{V}_{1}^{Q \bar{Q}} s \bar{s}\left(\begin{array}{cc}
-1 & 0 \\
0 & 1
\end{array}\right), \\
M_{1^{+-}} & =\left(M_{0}-\frac{4}{3} V_{8}\right)\left(\begin{array}{ll}
1 & 0 \\
0 & 1
\end{array}\right)+\tilde{V}_{2}^{Q \bar{Q}} s \bar{s}\left(\begin{array}{cc}
-1 & 0 \\
0 & 1
\end{array}\right), \\
M_{1^{++}} & =M_{0}-\kappa_{s Q}+\frac{4}{3} V_{8}, \\
M_{2^{++}} & =M_{0}+\kappa_{s Q}+\frac{4}{3} V_{8},
\end{aligned}
$$

where

$$
\begin{aligned}
& \tilde{V}_{1}^{Q \bar{Q} s \bar{s}} \equiv \sqrt{\kappa_{s Q}{ }^{2}-\frac{4}{3} \kappa_{s Q} V_{8}+\frac{16}{9} V_{8}^{2}}, \\
& \tilde{V}_{2}^{Q \bar{Q} s \bar{s}} \equiv \sqrt{\kappa_{s Q}{ }^{2}+\frac{64}{9} V_{8}^{2} .}
\end{aligned}
$$

\section{HIDDEN-CHARM, OPEN-STRANGE SECTOR}

In this sector, the Hamiltonian analogous to Eq. (8) becomes

$$
\begin{aligned}
H= & H_{0}+\Delta H_{\kappa_{q Q}}+\Delta H_{\kappa_{s Q}}+\Delta H_{V_{8}}, \\
= & H_{0}+2\left[\kappa_{q Q}\left(\mathbf{s}_{q} \cdot \boldsymbol{s}_{Q}\right)+\kappa_{s Q}\left(\mathbf{s}_{\bar{s}} \cdot s_{\bar{Q}}\right)\right] \\
& +V_{8}\left(\lambda_{a}^{8} \boldsymbol{\sigma}_{q}\right) \cdot\left(\lambda_{a}^{8} \boldsymbol{\sigma}_{\bar{s}}\right) .
\end{aligned}
$$

Without loss of generality, we have taken $q^{\prime} \rightarrow s$, with the opposite choice $q \rightarrow s$ simply leading to the antiparticles of those studied here. Then the spin couplings $\kappa_{s Q}$ and $\kappa_{q Q}$ are numerically quite distinct, and we compute the mass contributions

$$
\begin{aligned}
& \Delta M_{\kappa_{q Q}}=\frac{1}{2} \kappa_{q Q}\left[2 s_{\delta}\left(s_{\delta}+1\right)-3\right], \\
& \Delta M_{\kappa_{\bar{s} \bar{Q}}}=\frac{1}{2} \kappa_{s Q}\left[2 s_{\bar{\delta}}\left(s_{\bar{\delta}}+1\right)-3\right],
\end{aligned}
$$

and

$$
\Delta M_{V_{8}}=-\frac{2}{3} V_{8}\left[2 s_{q \bar{s}}\left(s_{q \bar{s}}+1\right)-3\right],
$$

with the final expression computed in the same manner as is performed to obtain Eq. (9).

A notable feature of the open-strange exotics sector becomes apparent when considering the full $\mathrm{SU}(3)_{\text {flavor }}$ 
multiplet structure. $Q \bar{Q} q \bar{q}$ states with $I_{3}=0$ carry good $J^{P C}$ quantum numbers, and states with different $J^{P C}$ values of course cannot mix with them. Inasmuch as isospin is a nearly exact symmetry, one can extend $C$ parity to a full isospin multiplet by defining the conserved $G$-parity quantum number (whose eigenvalues for all hadrons are tabulated by the Particle Data Group (PDG) [17]). Specifically,

$$
G \equiv(-1)^{I} C
$$

where the $C$ parity eigenvalue here is that of the $I_{3}=0$ member of the isomultiplet. One could generalize the concept of $G$ parity to a full $\mathrm{SU}(3)_{\text {flavor }}$ multiplet, but since the corresponding flavor symmetry is broken, mixing between the open-strange members of multiplets whose $I_{3}=0, Y=0$ members have opposite $C$ parities can occur. Indeed, this phenomenon is known among the conventional mesons: for example, the strange partners to the lightest $1^{++}$and $1^{+-}$mesons are named $K_{1 A}$ and $K_{1 B}$, respectively, and the observed $1^{+}$strangemeson mass eigenstates $K_{1}(1270)$ and $K_{1}(1400)$ are believed to be nearly equal admixtures of $K_{1 A}$ and $K_{1 B}$ [17].

In the exotics sector, the nonstrange $X_{1}\left(1^{++}\right)$states cannot mix with $\tilde{Z}, \tilde{Z}^{\prime}\left(1^{+-}\right)$due to $G$-parity conservation. However, their open-strange $1^{+}$partners can mix, leading to richer phenomenological possibilities. To wit: the mass expressions obtained from Eq. (17), prior to diagonalization, read

$$
\begin{aligned}
\tilde{M}_{0^{+}}= & M_{0}\left(\begin{array}{ll}
1 & 0 \\
0 & 1
\end{array}\right)-\frac{1}{2}\left(\kappa_{q Q}+\kappa_{s Q}\right)\left(\begin{array}{cc}
0 & \sqrt{3} \\
\sqrt{3} & 2
\end{array}\right) \\
& -\frac{2}{3} V_{8}\left(\begin{array}{cc}
-3 & 0 \\
0 & 1
\end{array}\right), \\
\tilde{M}_{1^{+}}= & M_{0}\left(\begin{array}{lll}
1 & 0 & 0 \\
0 & 1 & 0 \\
0 & 0 & 1
\end{array}\right)+\frac{\kappa_{q Q}}{2}\left(\begin{array}{ccc}
-1 & -\sqrt{2} & +\sqrt{2} \\
-\sqrt{2} & 0 & +1 \\
+\sqrt{2} & +1 & 0
\end{array}\right), \\
& +\frac{\kappa_{s Q}}{2}\left(\begin{array}{ccc}
-1 & +\sqrt{2} & -\sqrt{2} \\
+\sqrt{2} & 0 & +1 \\
-\sqrt{2} & +1 & 0
\end{array}\right)-\frac{2}{3} V_{8}\left(\begin{array}{ccc}
1 & 0 & 0 \\
0 & -3 & 0 \\
0 & 0 & 1
\end{array}\right), \\
M_{2^{+}}= & M_{0}+\frac{1}{2}\left(\kappa_{q Q}+\kappa_{s Q}\right)-\frac{2}{3} V_{8} .
\end{aligned}
$$

The elements of the matrices for $0^{+}$are again arranged in order of increasing heavy-quark spin. However, those for $1^{+}$are arranged in the order corresponding to increasing mass eigenvalues for their nonstrange partners in the hidden-charm sector: $X_{1}, \tilde{Z}^{\prime}, \tilde{Z}$.
The mass eigenvalues for the $0^{+}$sector read

$$
\begin{aligned}
M_{0^{+}}= & {\left[M_{0}-\frac{1}{2}\left(\kappa_{q Q}+\kappa_{s Q}\right)+\frac{2}{3} V_{8}\right]\left(\begin{array}{ll}
1 & 0 \\
0 & 1
\end{array}\right) } \\
& +2 \tilde{V}_{1}^{Q \bar{Q} q \bar{s}}\left(\begin{array}{cc}
-1 & 0 \\
0 & 1
\end{array}\right),
\end{aligned}
$$

where

$$
\tilde{V}_{1}^{Q \bar{Q} q \bar{s}} \equiv \sqrt{\left[\frac{1}{2}\left(\kappa_{q Q}+\kappa_{s Q}\right)+\frac{1}{3} V_{8}\right]^{2}+\frac{1}{3} V_{8}^{2}} .
$$

The exact expressions for the $1^{+}$eigenvalues are of course complicated roots of a cubic equation, but anticipating that $V_{8} \ll \kappa_{q Q} \ll \kappa_{s Q}$, one may perform a perturbative expansion in $V_{8}$ to compute approximate values:

$$
\begin{aligned}
& M_{1^{+}}^{(1)}=M_{0}^{Q \bar{Q} q \bar{s}}+\frac{1}{2}\left(-3 \kappa_{s Q}+\kappa_{q Q}\right)+O\left(V_{8}^{2} / \kappa_{s Q}\right), \\
& M_{1^{+}}^{(2)}=M_{0}^{Q \bar{Q} q \bar{s}}+\frac{1}{2}\left(\kappa_{s Q}-3 \kappa_{q Q}\right)+O\left(V_{8}^{2} / \kappa_{q Q}\right), \\
& M_{1^{+}}^{(3)}=M_{0}^{Q \bar{Q} q \bar{s}}+\frac{1}{2}\left(\kappa_{s Q}+\kappa_{q Q}\right)+\frac{2}{3} V_{8}+O\left(V_{8}^{2} / \kappa_{q Q}\right) .
\end{aligned}
$$

\section{ANALYSIS}

\section{A. Flavor SU(3) multiplets and mixing}

The original analysis of $c \bar{c} s \bar{s}$ states in Ref. [65], as well as its updated form in Ref. [62], takes the $c \bar{c} s \bar{s}$ states to be completely unmixed with those in the $c \bar{c} q \bar{q}^{\prime}$ sector, where $q, q^{\prime} \in\{u, d\}$. If, on the other hand, $\mathrm{SU}(3)_{\text {flavor }}$ is exact, then the states should fill octets and singlets of the flavor symmetry. Specifically, the flavor structure of $c \bar{c} q \bar{q}^{\prime}$ states, now allowing $q, q^{\prime} \in\{u, d, s\}$, can be discussed using the same framework that applies to conventional $q \bar{q}^{\prime}$ mesons. The $I=0, I_{3}=0, \quad Y=0$ unmixed octet and singlet combinations are, as usual,

$$
\frac{1}{\sqrt{6}}(u \bar{u}+d \bar{d}-2 s \bar{s}) \quad \text { and } \quad \frac{1}{\sqrt{3}}(u \bar{u}+d \bar{d}+s \bar{s}),
$$

respectively. In the lightest $\left(J^{P C}=0^{-+}\right)$meson multiplet, these states correspond to $\eta$ and $\eta^{\prime}$, respectively, which remain largely unmixed because the octet states are pseudoNambu-Goldstone bosons whose masses vanish in the chiral limit, while the singlet has a nonzero mass in this limit due to the anomalous breaking of the axial U(1) symmetry of massless QCD.

Heavier meson multiplets, however, support much larger $\mathrm{SU}(3)_{\text {flavor }}$ mixing between the octet and singlet combinations. For example, the next-lightest $\left(1^{--}\right)$multiplet features the $\omega$ and $\phi$ as its $I=0$ states, which appear to be 
nearly ideally mixed into the flavor combinations $\frac{1}{\sqrt{2}}(u \bar{u}+d \bar{d})$ and $s \bar{s}$, respectively. The appearance of only the $J / \psi \phi$ decay mode for most of the purported $c \bar{c} s \bar{s}$ candidates inspired the implicit adoption of an ideal-mixing ansatz in Refs. [62,65].

Moreover, the approach of treating $I=0$ states in the $c \bar{c} q \bar{q}^{\prime}$ sector as containing no $s \bar{s}$ component, which is implicit in Refs. [59,60,62], also introduces a hidden assumption of octet-singlet mixing into the analysis. The fact that $\rho^{0}(I=1$, pure octet $)$ and $\omega(I=0$, ideally mixed $)$ are nearly degenerate in mass, and likewise for $Z_{c}(3900)^{0}$ $(I=1$, pure octet) and $X(3872)(I=0)$, suggests that substantial octet-singlet flavor mixing is needed to understand the spectrum of both $1^{--}$conventional mesons and $\Sigma_{g}^{+}(1 S)$ hidden-charm exotic mesons. Isospin symmetry then links the remaining $Y=0$ states $\left(\rho^{ \pm}, Z_{c}(3900)^{ \pm}\right)$. In the case of exotics, the values of $M_{0}^{c \bar{c} q \bar{q}^{\prime}}$ and $M_{0}^{c \bar{c} s \bar{s}}$ extracted in previous work implicitly incorporate ideal octet-singlet flavor mixing, with strangeness dependence entering only through the differing diquark masses for $\delta=(c q)$ and for $\delta=(c s)$.

In contrast, the value of $M_{0}^{c \bar{c} q \bar{s}}$ for the open-strange ( $K$-like) states refers to an unmixed $\mathrm{SU}(3)_{\text {flavor }}$ octet. One should therefore not be surprised that simply combining the values of $m_{(c q)}$ from $M_{0}^{c \bar{c} q \bar{q}^{\prime}}$ and $m_{(c s)}$ from $M_{0}^{c \bar{c} s \bar{s}}$ with the lattice-computed glue potential $V(r)$ between heavy colortriplet sources generates a value for $M_{0}^{c \bar{c} q \bar{s}}$ slightly different from the one that would be obtained from starting with values of $M_{0}$ corresponding to unmixed $\mathrm{SU}(3)$-octet or -singlet $c \bar{c} q \bar{q}^{\prime}$ and $c \bar{c} s \bar{s}$ states. This effect should occur even in the absence of an explicit $\mathrm{SU}(3)_{\text {flavor }}$-breaking difference between $m_{\delta=(c q)}$ and $m_{\delta=(c s)}$.

We illustrate this mixing effect using a toy example well known from elementary quantum mechanics: ignore finestructure effects and let the unmixed mass parameters $M_{8}$ (pure octet) and $M_{1}$ (pure singlet) be degenerate, $M=M_{8}=M_{1}$, in a two-level system with an octet-singlet mass-mixing parameter $\Delta$. Then the resulting mass eigenvalues are $M \mp \Delta$, and the mixing angle of the system is maximal, $45^{\circ}$. More generally, the lower mass eigenvalue is always smaller than the smaller diagonal element, whether or not the unmixed octet and singlet mass parameters are equal. In our case, the value of $M_{0}^{c \bar{c} q \bar{q}^{\prime}}$ from the previous analyses of Refs. [59,60,62,65] is assumed to refer to ideally mixed states; and since one expects the exotics observed thus far (which are used to extract $M_{0}$ values) to be the lightest ones that exist, the derived $M_{0}^{c \bar{c} q \bar{q}^{\prime}}$ results represent the smaller mass eigenvalues. Meanwhile, $M_{0}^{c \bar{c} s \bar{s}}$ is extracted from states assumed to contain no light valence quarks, and therefore their component diquarks are pure $(c s)$; thus, no mixing needs to be performed to extract the parameter $m_{\delta=(c s)}$. The result for $M_{0}^{c \bar{c} q \bar{s}}$ naïvely obtained from using the (mixed) $M_{0}^{c \bar{c} q \bar{q}^{\prime}}$ and $M_{0}^{c \bar{c} s \bar{s}}$ values should therefore be slightly lower than one determined entirely from the pure-octet $c \bar{c} q \bar{s}$ sector. This expectation, in fact, is precisely what occurs, as we see below.

\section{B. $c \bar{c} q \bar{q}^{\prime}$ sector and $V_{\mathbf{8}}$}

The analysis of the $c \bar{c} q \bar{q}^{\prime}$ sector here closely follows that of Ref. [60], and especially Ref. [62]. The three primary inputs are the PDG averages [17]

$$
\begin{aligned}
m_{X(3872)} & =3871.69 \pm 0.17 \mathrm{MeV} \\
m_{Z_{c}(3900)} & =3888.4 \pm 2.5 \mathrm{MeV} \\
m_{Z_{c}(4020)} & =4024.1 \pm 1.9 \mathrm{MeV}
\end{aligned}
$$

with only the value for $Z_{c}(3900)$ changing slightly since the previous analyses. Since the Hamiltonian of Eq. (8) now has four parameters, the system is underdetermined. However, one further constraint arises from noting the strong charmonium decay preference [17] of $Z_{c}(3900)$ to $J / \psi$, and $Z_{c}(4020)$ to $h_{c}$, suggesting that these $Z_{c}$ states are nearly pure $s_{c \bar{c}}=1$ and $s_{c \bar{c}}=0$ eigenstates, respectively. Defining $P$ as the $s_{Q \bar{Q}}=1$ probability content of the lowermass $1^{+-}, I=1$ eigenstate of Eq. (12) [i.e., the square of the off-diagonal component of the unitary matrix diagonalizing $\tilde{M}_{1^{+-}}^{I=1}$ in Eq. (11)], one obtains

$$
P=\frac{1}{2}\left[1+\frac{2\left(V_{0}+\frac{V_{8}}{3}\right)}{\sqrt{\kappa_{q Q}{ }^{2}+4\left(V_{0}+\frac{V_{8}}{3}\right)^{2}}}\right],
$$

which means that $V_{8}$ can be expressed as a function of $P$ (and the parameters $V_{0}$ and $\kappa_{q Q}$ ). Using this constraint with the mass expressions in Eq. (12), the most convenient combinations of the three masses in Eq. (27) are

$$
\begin{aligned}
\mu_{1} & \equiv \frac{1}{2}\left(m_{Z_{c}(4020)}+m_{Z_{c}(3900)}\right)=3956.3 \pm 1.6 \mathrm{MeV}, \\
& =M_{0}-\frac{1}{2}\left(P-\frac{1}{2}\right) \frac{\kappa_{q c}}{\sqrt{P(1-P)}}, \\
\mu_{2} & \equiv \frac{1}{2}\left(m_{Z_{c}(4020)}+m_{Z_{c}(3900)}\right)-m_{X(3872)}, \\
& =84.6 \pm 1.6 \mathrm{MeV} \\
& =\kappa_{q c}\left[1-\frac{\left(P-\frac{1}{2}\right)}{\sqrt{P(1-P)}}\right]+4 V_{0}, \\
\mu_{3} & \equiv \frac{1}{2}\left(m_{Z_{c}(4020)}-m_{Z_{c}(3900)}\right)=67.9 \pm 1.6 \mathrm{MeV}, \\
& =\frac{\kappa_{q c}}{2 \sqrt{P(1-P)}} .
\end{aligned}
$$

From Eq. (29), one extracts 


$$
\frac{4}{3} V_{8}=-\mu_{2}+2 \mu_{3}\left[\left(P-\frac{1}{2}\right)+\sqrt{P(1-P)}\right] .
$$

The case $V_{8}=0$, which (in effect) is imposed in Ref. [62], becomes

$$
\begin{aligned}
P & =\frac{1}{4}\left[2+\frac{\mu_{2}}{\mu_{3}} \pm \sqrt{2-\left(\frac{\mu_{2}}{\mu_{3}}\right)^{2}}\right], \\
& =0.979 \pm 0.009,0.644 \pm 0.030
\end{aligned}
$$

The only input used in Ref. [62] beyond those of Eq. (27) is the discrete choice of the larger $P$ value in Eq. (31) to recognize the $Z_{c}$ charmonium decay preferences noted above.

In fact, Eq. (30) places a rather strong constraint upon $V_{8}$. While any $P \in[0,1]$ is in principle allowed, values of $P$ smaller (larger) than the smaller (larger) root in Eq. (31) lead to negative values of $V_{8}$-and, for sufficiently small values of $P$, values of $V_{8}$ that are also larger in magnitude than $V_{0}$. Inasmuch as the accompanying operators in Eq. (8) represent $\pi$-like and $\eta$-like exchanges, respectively, one expects the analogy to the dynamics of true $\pi$ and $\eta$ exchanges between nucleons (from, e.g., chiral perturbation theory) to hold. Under this assumption, the $\eta$-like exchange should be attractive like the $\pi$-like exchange; hence $V_{8}$, like $V_{0}$, should be positive. However, genuine $\eta$ exchange is also weaker than $\pi$ exchange, both due to the $\eta$ 's larger mass and larger decay constant. We therefore take $V_{8}>0$ to be a natural constraint of the model, which requires $P$ to lie between the roots given in Eq. (31). According to Eq. (30), within this range $V_{8}$ reaches a maximum at $P=\frac{1}{2}\left(1+\frac{1}{\sqrt{2}}\right)=0.854$, at which

$$
V_{8}^{\max }=\frac{3}{4}\left(-\mu_{2}+\sqrt{2} \mu_{3}\right)=8.6 \pm 2.3 \mathrm{MeV}
$$

We therefore expect the allowed range of $V_{8}$, as determined by the known phenomenology of the $c \bar{c} q \bar{q}^{\prime}$ sector, to have a modest effect compared to that provided by the other parameters in Eq. (8). Using Eq. (29), one obtains the following for the other Hamiltonian parameters:

$$
\begin{aligned}
M_{0} & =\mu_{1}+\left(P-\frac{1}{2}\right) \mu_{3}, \\
\kappa_{q c} & =2 \mu_{3} \sqrt{P(1-P)}, \\
4 V_{0} & =\mu_{2}+2 \mu_{3}\left[\left(P-\frac{1}{2}\right)-\sqrt{P(1-P)}\right] .
\end{aligned}
$$

The values of $M_{0}, \kappa_{q c}$, and $V_{0}$ obtained for both $V_{8}=0$ and for an optimized $V_{8}$ value obtained below from the $c \bar{c} s \bar{s}$ spectrum [in Eq. (45)] are presented in Table I. The
TABLE I. Hamiltonian parameters [Eq. (8)] of the dynamical diquark model obtained from fits to members of the $\Sigma_{g}^{+}(1 S)$ multiplet in the $c \bar{c} q \bar{q}^{\prime}\left[X(3872), Z_{c}(3900), Z_{c}(4020)\right]$ and $c \bar{c} s \bar{s}$ $[X(3915), X(4140), X(4350)]$ sectors. Also included is the $s_{c \bar{c}}=$ 1 content $P$ of the state $Z_{c}(3900)$, and the diquark mass $m_{\delta}$ derived from each case, as well as a value of $M_{0}^{c \bar{c} q \bar{s}}$ for the openstrange sector that supposes no $\mathrm{SU}(3)_{\text {flavor }}$ octet-singlet mixing in the other flavor sectors.

\begin{tabular}{lcc}
\hline \hline & $V_{8}=0$ & $V_{8}=3.9 \pm 1.4 \mathrm{MeV}$ \\
\hline$M_{0}^{c \bar{c} q \bar{q}^{\prime}}$ & $3988.7 \pm 1.5 \mathrm{MeV}$ & $3987.7 \pm 1.6 \mathrm{MeV}$ \\
$\kappa_{q c}$ & $19.6 \pm 3.7 \mathrm{MeV}$ & $25.6 \pm 5.0 \mathrm{MeV}$ \\
$V_{0}$ & $32.5 \pm 1.3 \mathrm{MeV}$ & $30.4 \pm 1.9 \mathrm{MeV}$ \\
$P$ & $0.979 \pm 0.009$ & $0.963 \pm 0.016$ \\
$m_{\delta(c q)}$ & $1927.0 \pm 11.5 \mathrm{MeV}$ & $1927.1 \pm 11.0 \mathrm{MeV}$ \\
$M_{0}^{c \bar{c} s \bar{s}}$ & $\ldots$ & $4251.3 \pm 2.8 \mathrm{MeV}$ \\
$\kappa_{s c}$ & $\ldots$ & $109.8 \pm 1.1 \mathrm{MeV}$ \\
$m_{\delta(c s)}$ & $\cdots$ & $2069.4 \pm 10.9 \mathrm{MeV}$ \\
$M_{0}^{c \bar{c} q \bar{s}}$ & $\cdots$ & $4119.7 \pm 1.7 \mathrm{MeV}$ \\
\hline \hline
\end{tabular}

full spectrum of masses for the $c \bar{c} q \bar{q}^{\prime} \Sigma_{g}^{+}(1 S)$ multiplet appears in Table II.

Using the value of $M_{0}^{c \bar{c} q \bar{q}^{\prime}}$ from Table I with $V_{8}=0$ and the lattice-simulated glue potentials $V(r)$ of Refs. [66,67] (labeled as JKM) and [68] (labeled as CPRRW), we compute

$$
\begin{array}{rll}
m_{\delta(c q)} & =1938.5 \pm 0.8 \mathrm{MeV} & (\mathrm{JKM}) \\
& =1915.5 \pm 0.8 \mathrm{MeV} & (\mathrm{CPRRW}) .
\end{array}
$$

A value spanning this spread is presented in Table I. Again, these results for $V_{8}=0$ differ from those in Ref. [62] only through a small shift in the tabulated PDG value of $m_{Z_{c}(3900)}$ in Eq. (27).

\section{C. $c \bar{c} s \bar{s}$ sector: $\kappa_{s c}$ and $V_{\mathbf{8}}$}

The signature process used in Ref. [62] to identify $c \bar{c} s \bar{s}$ exotics is the decay mode $J / \psi \phi$, although some candidates are identifiable through $D_{s}$-type meson-pair decays. Furthermore, the analysis of Ref. [62] argues that the $J^{P C}=$ $1^{++} X(4274)$ is an excellent candidate for the conventional charmonium state $\chi_{c 1}(3 P)$. The most unexpected addition to the $c \bar{c} s \bar{s}$ spectrum is the peculiar state $X(3915)$, with likely $0^{++}$quantum numbers, as the candidate for the lightest $c \bar{c} s \bar{s}$ state in this model. In brief summary of the reasoning in Ref. [65] and references therein, $X(3915)$ has no confirmed open-charm decays, thus arguing against it being either the conventional charmonium state $\chi_{c 0}(2 P)$ or $c \bar{c} q \bar{q}$. It has definitively been seen to couple only to $\gamma \gamma$ and $J / \psi \omega$; with respect to the latter mode, note that $X(3915)$ lies below the $J / \psi \phi$ threshold, so that $\phi \rightarrow \omega$ mixing is proposed in Ref. [65] to be responsible for the $J / \psi \omega$ decay mode. Furthermore, a recent lattice calculation [69] 
TABLE II. Predictions of hidden-charm plus light-quark tetraquark meson masses (in MeV) for all states in the lowest multiplet $\left[\Sigma_{g}^{+}(1 S)\right]$ of the dynamical diquark model, using the Hamiltonian parameters of Table I [and Eq. (54) for $c \bar{c} q \bar{s}$ ]. Observed masses (used to obtain the Hamiltonian parameters) are exhibited in boldface. Uncertainties are obtained by including those on all fit parameters.

\begin{tabular}{|c|c|c|c|c|c|c|c|c|c|}
\hline & \multicolumn{4}{|c|}{$c \bar{c} q \overline{q^{\prime}}$} & \multicolumn{2}{|c|}{$c \bar{c} s \bar{s}$} & \multicolumn{3}{|c|}{$c \bar{c} q \bar{s}$} \\
\hline \multirow{2}{*}{$\overline{\frac{J^{P C}}{0^{++}}}$} & \multicolumn{2}{|c|}{$I=0$} & \multicolumn{2}{|c|}{$I=1$} & & & \multicolumn{3}{|l|}{$J^{P}$} \\
\hline & $3841.9 \pm 13.0$ & $4262.1 \pm 17.1$ & $3872.1 \pm 9.6$ & $3988.7 \pm 2.3$ & $3921.7 \pm 4.3$ & $4350.9 \pm 4.7$ & $0^{+}$ & $3951.8 \pm 7.7$ & $4228.0 \pm 10.4$ \\
\hline $1^{+-}$ & $3896.0 \pm 5.9$ & $4259.2 \pm 17.1$ & $3887.2 \pm 6.1$ & $4024.4 \pm 3.0$ & $4135.8 \pm 3.7$ & $4356.4 \pm 3.4$ & $1^{+}$ & $4003.0 \pm 7.6$ & $4171.3 \pm 10.3$ \\
\hline $1^{++}$ & $\mathbf{3 8 7 2 . 2} \pm 7.8$ & $\ldots$ & $3993.8 \pm 5.6$ & $\ldots$ & $4146.7 \pm 3.5$ & $\ldots$ & & $4225.6 \pm 7.5$ & $\ldots$ \\
\hline $2^{++}$ & $3923.4 \pm 7.8$ & $\ldots$ & $4045.0 \pm 5.6$ & $\ldots$ & $4366.3 \pm 3.5$ & $\ldots$ & $2^{+}$ & $4220.1 \pm 7.5$ & $\ldots$ \\
\hline
\end{tabular}

predicts the existence of a $0^{++}$state in this mass region that has a strong coupling to $D_{s} \bar{D}_{s}$ but a weak coupling to $D \bar{D}$. The mass used in this work is the PDG value [17]:

$$
m_{X(3915)}=3921.7 \pm 1.8 \mathrm{MeV}
$$

In the previous analysis [62], the $c \bar{c} s \bar{s}$ spectrum obtained for the multiplet is very simple. Referring to Eq. (15), the assumption that $\kappa_{s c} \gg V_{8}>0$ leads to the spectrum (in increasing order of mass):

$$
\begin{aligned}
& M_{0^{++}}=M_{0}-3 \kappa_{s c}+O\left(V_{8}^{2} / \kappa_{s c}\right), \\
& M_{1^{+-}}=M_{0}-\kappa_{s c}-\frac{4}{3} V_{8}+O\left(V_{8}^{2} / \kappa_{s c}\right), \\
& M_{1^{++}}=M_{0}-\kappa_{s c}+\frac{4}{3} V_{8}, \\
& M_{0^{++}}^{\prime}=M_{0}+\kappa_{s c}-\frac{8}{3} V_{8}+O\left(V_{8}^{2} / \kappa_{s c}\right), \\
& M_{1^{+-}}^{\prime}=M_{0}+\kappa_{s c}-\frac{4}{3} V_{8}+O\left(V_{8}^{2} / \kappa_{s c}\right), \\
& M_{2^{++}}=M_{0}+\kappa_{s c}+\frac{4}{3} V_{8},
\end{aligned}
$$

which reduces to three degenerate sets in the case $V_{8}=0$, as listed in Ref. [62]. In particular, the lighter $0^{++}$state clearly lies far below the others, with the $1^{++}$state (and the lighter $1^{+-}$) being intermediate in mass, and the $2^{++}$and heavier $0^{++}\left(\right.$and $1^{+-}$) states lying close together at a larger mass value. The overall effect of Eq. (36) is to split the $\Sigma_{g}^{+}(1 S)$ multiplet into three roughly equally spaced (by $2 \kappa_{s c}$ ) clusters of $c \bar{c} s \bar{s}$ states.

The state $X(4140)$ is taken to be an unmistakable $c \bar{c} s \bar{s}$ candidate, the sole $1^{++}$member of the multiplet $\Sigma_{g}^{+}(1 S)$. Therefore, the $c \bar{c} s \bar{s}$ spectrum should start with $X(3915)$ being the distinct lightest member, a $1^{+-}$state is predicted to appear with a mass near $m_{X(4140)}$, and a trio of states $\left(0^{++}\right.$, $\left.1^{+-}, 2^{++}\right)$is predicted to appear at approximately $m_{X(3915)}+2\left(m_{X(4140)}-m_{X(3915)}\right)$. A complication arises, however, with the latest LHCb measurement [5] of $X(4140)$ :

$$
\begin{aligned}
m_{X(4140)} & =4118 \pm 11_{-36}^{+19} \mathrm{MeV}, \\
\Gamma_{X(4140)} & =162 \pm 21_{-49}^{+24} \mathrm{MeV},
\end{aligned}
$$

which should be compared to the PDG average [17],

$$
\begin{aligned}
m_{X(4140)} & =4146.8 \pm 2.4 \mathrm{MeV}, \\
\Gamma_{X(4140)} & =22_{-7}^{+8} \mathrm{MeV},
\end{aligned}
$$

the mass differing by about $1.3 \sigma$ (and the width differing radically). LHCb observes $X(4140)$ with a $13 \sigma$ total significance. On the other hand, the previous LHCb observation of $X(4140)$ [70] (at a significance of $8.4 \sigma$ ) forms part of the PDG averages of Eq. (38), and the mass value obtained in Ref. [70] is much more in line with the average mass value given in Eq. (38):

$$
\begin{aligned}
m_{X(4140)} & =4146.5 \pm 4.5_{-2.8}^{+4.6} \mathrm{MeV}, \\
\Gamma_{X(4140)} & =83 \pm 21_{-14}^{+21} \mathrm{MeV} .
\end{aligned}
$$

In fact, the data used in Ref. [70] forms a small subset of the $\mathrm{LHCb}$ data reported in Ref. [5]. So how can a measurement using much more data lead to a result with much larger uncertainties? In large part, it arises from a new modeling of the $X(4140)$ line shape, in which a naïve Breit-Wigner profile is replaced with a Flatté form [71]. To incorporate this new development, we reanalyze the PDG mass average of Eq. (38) by replacing the old $\mathrm{LHCb}$ mass measurement of Eq. (39) with the new one of Eq. (37), producing the value to be used in our analysis:

$$
m_{X(4140)}=4146.7 \pm 2.7 \mathrm{MeV} .
$$

The state $X(4350)$, although not yet confirmed at the same level of confidence $(3.2 \sigma)$, is seen in $\gamma \gamma \rightarrow J / \psi \phi$ and thus is an excellent $c \bar{c} s \bar{s} 0^{++}$or $2^{++}$candidate. Noting that [17]

$$
m_{X(4350)}=4351 \pm 5 \mathrm{MeV},
$$

and using Eqs. (35) and (40), one finds 
$m_{X(3915)}+2\left(m_{X(4140)}-m_{X(3915)}\right)=4371.7 \pm 5.7 \mathrm{MeV}$.

One then sees (as in Refs. [62,65]) that $X(4350)$ nearly satisfies the equal-spacing rule discussed above, which confirms our previous result that $V_{8}$ is numerically small. In fact, at linear order in $V_{8}$, Eq. (36) gives

$$
\begin{aligned}
& \left(M_{0^{++}}^{\prime}-M_{1^{++}}\right)-\left(M_{1^{++}}-M_{0^{++}}\right)=-\frac{16}{3} V_{8}, \\
& \left(M_{2^{++}}-M_{1^{++}}\right)-\left(M_{1^{++}}-M_{0^{++}}\right)=-\frac{4}{3} V_{8} .
\end{aligned}
$$

Using $m_{X(4350)}$ from Eq. (41) for $M_{0^{++}}^{\prime}$ or $M_{2^{++}}$gives

$$
\begin{aligned}
& \left(m_{X(4350)}-m_{X(4140)}\right)-\left(m_{X(4140)}-m_{X(3915)}\right) \\
& \quad=-20.7 \pm 7.6 \mathrm{MeV}
\end{aligned}
$$

meaning that $V_{8}$ is small and positive, as anticipated in Eq. (32). Returning to the full mass expressions of Eq. (15), one uses Eqs. (35), (40), and (41) to obtain

$$
\begin{aligned}
M_{0}^{c \bar{c} s \bar{s}} & =4251.3 \pm 2.8 \mathrm{MeV}, \\
\kappa_{s c} & =109.8 \pm 1.1 \mathrm{MeV}, \\
V_{8} & =3.9 \pm 1.4 \mathrm{MeV},
\end{aligned}
$$

assuming that $X(4350)$ is $0^{++}$, and

$$
\begin{aligned}
M_{0}^{c \bar{c} s \bar{s}} & =4230.8 \pm 7.0 \mathrm{MeV}, \\
\kappa_{s c} & =102.2 \pm 2.8 \mathrm{MeV}, \\
V_{8} & =13.6 \pm 4.3 \mathrm{MeV},
\end{aligned}
$$

assuming that $X(4350)$ is $2^{++}$. Obtaining these results requires the resolution of a discrete ambiguity to impose the physical expectation $\kappa_{s c}>0$, as discussed in Ref. [62]. The latter solution produces a slightly larger value of $V_{8}$ than allowed by Eq. (32), but only by $1.0 \sigma$, and therefore still viable. Nevertheless, for purposes of illustration, we choose Eq. (45) as the best-fit parameters (also included in Table I), and use them to compute the full spectrum of masses for the $\Sigma_{g}^{+}(1 S) c \bar{c} s \bar{s}$ multiplet in Table II. In particular, using the value of $M_{0}^{c \bar{c} s \bar{s}}$ from Eq. (45) and the lattice-simulated glue potentials $V(r)$ of Refs. [66-68], we compute

$$
\begin{array}{rll}
m_{\delta(c s)} & =2080.2 \pm 1.5 \mathrm{MeV} & (\mathrm{JKM}), \\
& =2058.5 \pm 1.5 \mathrm{MeV} \quad(\mathrm{CPRRW}) .
\end{array}
$$

The $M_{0}^{c \bar{c} s \bar{s}}$ and $\kappa_{s c}$ values obtained in Eqs. (45) and (47) differ rather little from those in Ref. [62], in part because the previous work effectively takes $V_{8}=0$, and also because the inputs of Eqs. (35) and (40) have changed little in the interim.
Feeding the value of nonzero $V_{8}$ back into the $c \bar{c} q \bar{q}^{\prime}$ expressions given by Eqs. (30) and (33), one obtains the $V_{8}>0$ values of $M_{0}^{c \bar{c} q \bar{q}^{\prime}}, \kappa_{q c}, V_{0}$, and $P$ given in Table I. Using this value of $M_{0}^{c \bar{c} q \bar{q}^{\prime}}$ and the lattice-simulated glue potentials $V(r)$ of Refs. [66-68], we compute

$$
\begin{array}{rll}
m_{\delta(c q)} & =1938.0 \pm 0.9 \mathrm{MeV} & (\mathrm{JKM}) \\
& =1916.2 \pm 0.9 \mathrm{MeV} & (\mathrm{CPRRW})
\end{array}
$$

The averaged values for Eqs. (47) and (48) appear in Table I.

\section{D. $c \bar{c} q \overline{\boldsymbol{s}}$ sector}

The results obtained from the $c \bar{c} q \bar{q}^{\prime}$ and $c \bar{c} s \bar{s} \Sigma_{g}^{+}(1 S)$ multiplets in the previous two subsections, with parameters collected in Table I, are almost completely sufficient to predict the entire $c \bar{c} q \bar{s} \Sigma_{g}^{+}(1 S)$ spectrum. However, as noted in Sec. VA, the fact that the open-strange states are pure $\mathrm{SU}(3)_{\text {flavor }}$ octet, while $c \bar{c} q \bar{q}^{\prime}$ and $c \bar{c} s \bar{s}$ are assumed to be ideally mixed octet-singlet combinations, means that the value of $M_{0}^{c \bar{c} q \bar{s}}$ extracted using only inputs from the other sectors is likely to be slightly too low to match observed $Z_{c s}$ masses in Eq. (2). On the other hand, the fine structure obtained in this sector using values of $\kappa_{s c}$, $\kappa_{s q}$, and $V_{8}$ from Table I should be predicted correctly.

Explicitly, using the $m_{\delta(c q)}$ from Eq. (48), $m_{\delta(c s)}$ from Eq. (47), and the same lattice-calculated potentials $V(r)$ as used previously, we compute

$$
\begin{array}{rll}
M_{0}^{c \bar{c} q \bar{s}} & =4119.7 \pm 1.7 \mathrm{MeV} & (\mathrm{JKM}) \\
& =4119.7 \pm 1.7 \mathrm{MeV} & (\mathrm{CPRRW})
\end{array}
$$

a remarkably stable result across simulations. Using this value along with the other parameters in Table $\mathrm{I}$ in the $1^{+}$ expressions of Eq. (22), we compute

$$
\begin{aligned}
& m_{Z_{c s}^{(1)}}=3967.7 \pm 3.4 \mathrm{MeV}, \\
& m_{Z_{c s}^{(2)}}=4136.0 \pm 7.1 \mathrm{MeV}, \\
& m_{Z_{c s}^{(3)}}=4190.3 \pm 3.1 \mathrm{MeV},
\end{aligned}
$$

which are lower than the measured values given in Eq. (2). If, however, we add an offset

$$
\Delta M_{0}^{c \bar{c} q \bar{s}}=35.3 \pm 6.9 \mathrm{MeV}
$$

then the predictions of Eq. (50) becomes

$$
\begin{aligned}
& m_{Z_{c s}^{(1)}}=4003.0 \pm 7.6 \mathrm{MeV}, \\
& m_{Z_{c s}^{(2)}}=4171.3 \pm 10.3 \mathrm{MeV}, \\
& m_{Z_{c s}^{(3)}}=4225.6 \pm 7.5 \mathrm{MeV},
\end{aligned}
$$


and so $m_{Z_{c s}^{(1)}}$ and $m_{Z_{c s}^{(3)}}$ beautifully match the observed values in Eq. (2).

Of course, this model predicts also a third open-strange $1^{+}$state $Z_{c s}^{(2)}$, which is not, as yet, reported by LHCb. In this regard, we note that the reported mass uncertainty and width of $Z_{c s}(4220)$ in Eq. (2) are quite large, meaning that subsequent analysis might resolve the peak as two states $Z_{c s}^{(2)}$ and $Z_{c s}^{(3)}$, as was found for the pentaquark candidates $P_{c}(4440)$ and $P_{c}(4457)$ in Ref. [72]. Small hints of additional structure may be already visible in the $\mathrm{LHCb}$ results above $4100 \mathrm{MeV}$ (Fig. 3 of [72], right inset). The relative closeness of $Z_{c s}^{(2)}$ and $Z_{c s}^{(3)}$ in mass follows directly in this model, as can be seen from Eq. (25), since (Table I) $\kappa_{q c} \ll \kappa_{s c}$. The large mass splitting $m_{Z_{c s}^{(3)}}-m_{Z_{c s}^{(1)}} \approx 2 \kappa_{s c}>$ $200 \mathrm{MeV}$ is also explained naturally by the model; in this regard, note that such a large mixing would not have occurred without the mixing of strange states between $1^{++}$ and $1^{+-}$multiplets, as discussed in Sec. IV.

One other criterion may be useful for disentangling the trio of $1^{+}$states $Z_{c s}^{(i)}$ : the eigenvectors of Eq. (22) couple differently to the heavy- and light-quark-spin eigenstates $X_{1}$ [Eq. (6)], $\tilde{Z}^{\prime}$, and $\tilde{Z}$ [defined in Eq. (7)]. Explicitly, in the limit $V_{8}=0$, the corresponding eigenvectors with respect to this basis are

$v^{(1),(2),(3)}=\frac{1}{2}\left(\begin{array}{c}+\sqrt{2} \\ -1 \\ +1\end{array}\right), \frac{1}{2}\left(\begin{array}{c}-\sqrt{2} \\ -1 \\ +1\end{array}\right), \frac{1}{\sqrt{2}}\left(\begin{array}{c}0 \\ +1 \\ +1\end{array}\right)$.

Since only $\tilde{Z}$ has $s_{Q \bar{Q}}=0$, the third component of each eigenvector indicates the relative strength of the coupling to $h_{c}$ (vs $J / \psi$ ) in decays that conserve heavy-quark spin. We note that $Z_{c s}^{(2)}$ has the largest such coupling: $50 \%$ of its decays should be to $s_{Q \bar{Q}}=0$ states. Therefore, a prediction of this model is not only that $Z_{c s}(4220)$ resolves into two peaks, but also that the lower state couples particularly strongly to $h_{c}$.

Using the values of $M_{0}^{c \bar{c} q \bar{s}}, \kappa_{s c}, \kappa_{q c}$, and $V_{8}$ from Table I and the shifted multiplet average mass [using Eq. (51)],

$$
\tilde{M}_{0}^{c \bar{c} q \bar{s}} \equiv M_{0}^{c \bar{c} q \bar{s}}+\Delta M_{0}^{c \bar{c} q \bar{s}}=4155.0 \pm 7.5 \mathrm{MeV},
$$

we tabulate mass values for all members of the $\Sigma_{g}^{+}(1 S)$ $c \bar{c} q \bar{s}$ multiplet in Table II.

\section{E. The $c \bar{c} u d s$ pentaquark}

The numerical analysis of Ref. [59] builds upon the proposal of pentaquarks as diquark-triquark bound states [57], where the triquark $\bar{\theta}$ is formed using a concatenation of the same color-triplet-binding mechanism as appears within the diquarks:

$$
\bar{\theta} \equiv\left[\bar{Q}\left(q_{1} q_{2}\right) \overline{\mathbf{3}}\right]_{\mathbf{3}} .
$$

The dynamical diquark model then uses the same latticecalculated potentials as before [66-68] to connect the diquark and triquark quasiparticles. In the original calculations of Ref. [59], the pentaquark candidates $P_{c}$ (4312), $P_{c}$ (4380), $P_{c}(4440)$, and $P_{c}(4457)$ observed at $\mathrm{LHCb}$ $[72,73]$ are considered as $\delta=(c u), \bar{\theta}=[\bar{c}(u d)]$ bound states using a coarse analysis: LHCb identifies $P_{c}(4380)$ as having opposite parity to $P_{c}(4440) / P_{c}(4457)[73]{ }^{3}$ and such a small splitting between multiplets of opposite parity in the model makes sense only if $P_{c}(4380)$ is a high-lying state in the $P=-$ multiplet $^{4} \Sigma^{+}(1 S)$ $\left[J^{P}=\left(\frac{1}{2}, \frac{3}{2}\right)^{-}\right]$, while the other states belong to the $P=+$ multiplet $\Sigma^{+}(1 P)\left[J^{P}=\left(\frac{1}{2}, \frac{3}{2}, \frac{5}{2}\right)^{+}\right]$.

Using $P_{c}(4312)$ to fix $M_{0}$ for the $\Sigma^{+}(1 P)$ multiplet, where [72]

$$
m_{P_{c}(4312)}=4311.9 \pm 6.8 \mathrm{MeV},
$$

and using $m_{\delta=(c q)}$ obtained from the $c \bar{c} q \bar{q}^{\prime}$ states, Ref. [59] computes a value of $m_{\bar{\theta}} \simeq 1.93 \mathrm{GeV}$. Repeating the analysis here using the new value of $m_{\delta=(c q)}$ from Eq. (48), we find

$$
\begin{array}{rll}
m_{\bar{\theta}=\bar{c}\left(q_{1} q_{2}\right)} & =1884.6 \pm 7.5 \mathrm{MeV} & (\mathrm{JKM}), \\
& =1866.5 \pm 7.5 \mathrm{MeV} \quad(\mathrm{CPRRW}) .
\end{array}
$$

As noted in Ref. [59], $m_{\delta=(c q)}$ and $m_{\bar{\theta}=\left[\bar{c}\left(q_{1} q_{2}\right)\right]}$ are quite close in mass; indeed, $m_{\bar{\theta}}$ is actually slightly smaller than $m_{\delta}$ in the new calculation. This peculiarity arises from assigning the lowest observed $c \bar{c} q \bar{q}^{\prime}$ states to the groundstate $\Sigma_{g}^{+}(1 S)$ multiplet but assigning the lowest observed pentaquark $P_{c}(4312)$ to the excited $\Sigma^{+}(1 P)$ multiplet. Should the opposite-parity $P_{c}(4380)$ disappear from future data, then $P_{c}(4312)$ and the other states would become suitable to belong to $\Sigma^{+}(1 S)$, and $m_{\bar{\theta}}$ (absorbing what was orbital excitation energy) would become numerically larger. Even in the current circumstance, however, no obvious physical requirement demands that $m_{\bar{\theta}}>m_{\delta}$. Indeed, one may argue that $\bar{\theta}$ contains two significant sources of binding energy: within the diquark $\left(q_{1} q_{2}\right)$, and between this diquark and $\bar{c}$, while $\delta$ possesses only the first type of binding, thereby allowing $m_{\bar{\theta}} \lesssim m_{\delta}$.

Now suppose that the state $P_{c s}(4459)$ of Eq. (3) is the open-strange $\Sigma^{+}(1 P)$ analog to $P_{c}(4312)$, i.e., a $\delta-\bar{\theta}$ state with $\delta=(c s)$, where $m_{\delta=(c s)}$ is given in Eq. (47). Then we compute

\footnotetext{
${ }^{3}$ It is actually the original, unresolved $P_{c}(4450)$ of Ref. [73] that has opposite parity to $P_{c}(4380)$, and an assumption of this work that the two resolved components $P_{c}(4440)$ and $P_{c}(4457)$ [72] share this same parity eigenvalue.

${ }^{4}$ The $g$ quantum number is lost in the asymmetric diquarktriquark case [58].
} 


$$
\begin{array}{rll}
M_{0}^{c \bar{c} s q_{1} q_{2}} & =4441.8 \pm 7.0 \mathrm{MeV} & (\mathrm{JKM}) \\
& =4441.7 \pm 7.0 \mathrm{MeV} & (\mathrm{CPRRW})
\end{array}
$$

This is a stunning result, being less than $2 \sigma$ lower than the value in Eq. (3). Indeed, no reason apart from convenience leads one to take the $P_{c}(4312)$ [as opposed to, say, $\left.P_{c}(4457)\right]$ and $P_{c s}(4459)$ masses equal to the $M_{0}$ values for their respective $\Sigma^{+}(1 P)$ multiplets, except that they are the lightest ones known. A complete analysis would incorporate fine structure, as is done for the tetraquark sectors, but this exercise has not yet been carried out in the pentaquark sectors of this model, due to a lack of experimental clarity on $J^{P}$ quantum numbers for at least some of the observed states. Nevertheless, the result of Eq. (58) shows that a single model can, in fact, accommodate exotics in all observed flavor sectors.

The development of the dynamical diquark model to date has focused primarily on spectroscopy, and to a lesser extent on identifying the dominant quarkonium decay channels. Detailed quantitative calculations of strong decay widths, particularly for open-heavy-flavor channels, have not yet been attempted, because a precise description of couplings between diquark and hadron-hadron configurations has not yet been developed. Qualitative statements to explain the relative narrowness of exotic states have appeared since the initial description of the picture in Refs. [56,57]; they originate from the significant spatial separation between the diquark or triquark quasiparticles, which hinders the rearrangement of their component quarks into color-singlet hadrons. Such effects may need to be quite potent in the pentaquarks, since $P_{c}(4312), P_{c}(4440)$, $P_{c}(4457), P_{c s}(4459)$ all have surprisingly small widths $(\lesssim 20 \mathrm{MeV})$. In addition, the proximity of these states to $\bar{D}^{(*)} \Sigma_{c}^{(*)}$ or $\bar{D}^{(*)} \Xi_{c}$ thresholds has been noted since the original experimental papers, and predicted earlier in molecular models [74-77]. The next phase of the development of the model will address the effect of mixing between diquark configurations and hadron-hadron thresholds, thus providing critical insight into both the decay properties of exotics and a connection to the successes of hadronicmolecule pictures.

\section{CONCLUSIONS}

This work shows that the newly observed hiddencharm, open-strange exotic-hadron candidates $Z_{c s}(4000)$, $Z_{c s}(4220)$, and $P_{c s}(4459)$ fit naturally into the dynamical diquark model. Notably, the same lattice-simulated potential $V(r)$ between two heavy, color-triplet sources in the lowest Born-Oppenheimer configuration $\Sigma_{(g)}^{+}$is seen to apply to all cases studied here.

Among tetraquarks, the same Hamiltonian parameters, with numerical values obtained from the $c \bar{c} q \bar{q}^{\prime}\left(q, q^{\prime}\right.$ being $u$ or $d$ ) and $c \bar{c} s \bar{s}$ exotic $\Sigma_{g}^{+}(1 S)$ multiplets, successfully predict masses in the $c \bar{c} q \bar{s}$ sector. In particular, the large $Z_{c s}(4220)-Z_{c s}(4000)$ mass splitting emerges naturally as consequences of both the large (cs) diquark internal spinspin coupling $\kappa_{s c}$ and the mixing of open-strange members of $J^{P C}=1^{++}$and $1^{+-}$multiplets, the latter an effect seen in conventional hadron physics for strange mesons such as $K_{1 A}$ and $K_{1 B}$. The model also predicts a third $1^{+} Z_{c s}$ state lying not far below $4200 \mathrm{MeV}$.

The overall multiplet-average mass $M_{0}$ for $c \bar{c} q \bar{s}$ states also receives a shift modification compared to those for $c \bar{c} q \bar{q}^{\prime}$ and $c \bar{c} s \bar{s}$ states, since the former are pure $\mathrm{SU}(3)_{\text {flavor }}$ octet states, while states in the latter sets are assumed in the numerical analysis to be ideally mixed octet-singlet combinations. The size of this shift $\Delta M_{0}^{c \bar{c} q \bar{s}}$ is found to be numerically not large, at most a few 10 's of $\mathrm{MeV}$.

In addition, the model in all sectors has been expanded to allow not only a $\pi$-like interaction operator between the diquarks (as in previous studies), but an $\eta$-like interaction operator as well. The numerical size of the coefficient $V_{8}$ of this operator is found to be much smaller than that $\left(V_{0}\right)$ for $\pi$-like interactions, and has a fairly minimal effect on the hadron spectra. Mass predictions for all states in the $\Sigma_{g}^{+}(1 S)$ multiplet for each flavor content are presented.

Among pentaquarks, a crude calculation taking the nonstrange $P_{c}(4312)$ as a base state for the positive-parity multiplet $\Sigma^{+}(1 P)$ constructed of a diquark-triquark pair $(c q)[\bar{c}(u d)]$, and replacing the $(c q)$ diquark with a $(c s)$ diquark, produces a state very close in mass to that of $P_{c s}(4459)$.

As new exotic hadrons continue to be uncovered-a rather safe expectation, considering the rate of observational advances over the past few years-more opportunities for sharpening our understanding of their mass spectrum and transitions will emerge. Whether or not a diquark-based spectrum provides the eventual global picture for these states, the dynamical diquark model supplies a definite road map for the sort of spectrum to expect.

\section{ACKNOWLEDGMENTS}

This work was supported by the National Science Foundation (NSF) under Grant No. PHY-1803912. We thank T. Skwarnicki for helpful insight into the LHCb results. 
[1] R. Aaij et al. (LHCb Collaboration), Phys. Rev. Lett. 125, 242001 (2020).

[2] R. Aaij et al. (LHCb Collaboration), Phys. Rev. D 102, 112003 (2020).

[3] R. Aaij et al. (LHCb Collaboration), Sci. Bull. 66, 1391 (2021); 66, 1278 (2021).

[4] M. Ablikim et al. (BESIII Collaboration), Phys. Rev. Lett. 126, 102001 (2021).

[5] R. Aaij et al. (LHCb Collaboration), arXiv:2103.01803.

[6] R. Lebed, R. Mitchell, and E. Swanson, Prog. Part. Nucl. Phys. 93, 143 (2017).

[7] H.-X. Chen, W. Chen, X. Liu, and S.-L. Zhu, Phys. Rep. 639, 1 (2016).

[8] A. Hosaka, T. Iijima, K. Miyabayashi, Y. Sakai, and S. Yasui, Prog. Theor. Exp. Phys. 2016, 062C01 (2016).

[9] A. Esposito, A. Pilloni, and A. Polosa, Phys. Rep. 668, 1 (2017).

[10] F.-K. Guo, C. Hanhart, U.-G. Meißner, Q. Wang, Q. Zhao, and B.-S. Zou, Rev. Mod. Phys. 90, 015004 (2018).

[11] A. Ali, J. Lange, and S. Stone, Prog. Part. Nucl. Phys. 97, 123 (2017).

[12] S. Olsen, T. Skwarnicki, and D. Zieminska, Rev. Mod. Phys. 90, 015003 (2018).

[13] M. Karliner, J. Rosner, and T. Skwarnicki, Annu. Rev. Nucl. Part. Sci. 68, 17 (2018).

[14] C.-Z. Yuan, Int. J. Mod. Phys. A 33, 1830018 (2018).

[15] Y.-R. Liu, H.-X. Chen, W. Chen, X. Liu, and S.-L. Zhu, Prog. Part. Nucl. Phys. 107, 237 (2019).

[16] N. Brambilla, S. Eidelman, C. Hanhart, A. Nefediev, C.-P. Shen, C. Thomas, A. Vairo, and C.-Z. Yuan, Phys. Rep. 873, 1 (2020).

[17] P. Zyla et al. (Particle Data Group), Prog. Theor. Exp. Phys. 2020, 083C01 (2020).

[18] L. Maiani, A. Polosa, and V. Riquer, Sci. Bull. 66, 1616 (2021).

[19] S. Lee, M. Nielsen, and U. Wiedner, J. Korean Phys. Soc. 55, 424 (2009).

[20] D. Ebert, R. Faustov, and V. Galkin, Eur. Phys. J. C 58, 399 (2008).

[21] D.-Y. Chen, X. Liu, and T. Matsuki, Phys. Rev. Lett. 110, 232001 (2013).

[22] J. Dias, X. Liu, and M. Nielsen, Phys. Rev. D 88, 096014 (2013).

[23] M. Voloshin, Phys. Lett. B 798, 135022 (2019).

[24] J. Ferretti and E. Santopinto, J. High Energy Phys. 04 (2020) 119.

[25] J.-Z. Wang, Q.-S. Zhou, X. Liu, and T. Matsuki, Eur. Phys. J. C 81, 51 (2021).

[26] Z. Yang, X. Cao, F.-K. Guo, J. Nieves, and M. Pavon Valderrama, Phys. Rev. D 103, 074029 (2021).

[27] L. Meng, B. Wang, and S.-L. Zhu, Phys. Rev. D 102, 111502 (2020).

[28] M.-Z. Liu, J.-X. Lu, T.-W. Wu, J.-J. Xie, and L.-S. Geng, arXiv:2011.08720.

[29] B.-D. Wan and C.-F. Qiao, Nucl. Phys. B968, 115450 (2021).

[30] R. Chen and Q. Huang, Phys. Rev. D 103, 034008 (2021).

[31] M.-C. Du, Q. Wang, and Q. Zhao, arXiv:2011.09225.
[32] X. Cao, J.-P. Dai, and Z. Yang, Eur. Phys. J. C 81, 184 (2021).

[33] Z.-F. Sun and C.-W. Xiao, arXiv:2011.09404.

[34] Q.-N. Wang, W. Chen, and H.-X. Chen, arXiv:2011.10495.

[35] B. Wang, L. Meng, and S.-L. Zhu, Phys. Rev. D 103, L021501 (2021).

[36] Z.-G. Wang, Chin. Phys. C 45, 073107 (2021).

[37] K. Azizi and N. Er, Eur. Phys. J. C 81, 61 (2021).

[38] X. Jin, X. Liu, Y. Xue, H. Huang, and J. Ping, arXiv:2011.12230.

[39] J. Y. Süngü, A. Türkan, H. Sundu, and E. Veliev, arXiv: 2011.13013.

[40] N. Ikeno, R. Molina, and E. Oset, Phys. Lett. B 814, 136120 (2021).

[41] Y.-J. Xu, Y.-L. Liu, C.-Y. Cui, and M.-Q. Huang, arXiv: 2011.14313.

[42] L. Meng, B. Wang, and S.-L. Zhu, Sci. Bull. 66, 1288 (2021); 66, 1288 (2021).

[43] Z.-G. Wang, Int. J. Mod. Phys. A 36, 2150107 (2021).

[44] Z.-H. Guo and J. Oller, Phys. Rev. D 103, 054021 (2021).

[45] R. Albuquerque, S. Narison, and D. Rabetiarivony, Phys. Rev. D 103, 074015 (2021).

[46] U. Özdem and K. Azizi, arXiv:2102.09231.

[47] M.-J. Yan, F.-Z. Peng, M. Sánchez Sánchez, and M. Pavon Valderrama, arXiv:2102.13058.

[48] Y.-H. Ge, X.-H. Liu, and H.-W. Ke, arXiv:2103.05282.

[49] X. Chen, Y. Tan, and Y. Chen, Phys. Rev. D 104, 014017 (2021).

[50] P. Ortega, D. Entem, and F. Fernández, Phys. Lett. B 818, 136382 (2021).

[51] H.-X. Chen, arXiv:2103.08586.

[52] L. Maiani, A. Polosa, and V. Riquer, Symmetry 13, 751 (2021).

[53] L. Meng, G.-J. Wang, B. Wang, and S.-L. Zhu, arXiv:2104 .08469 .

[54] U. Özdem and A. Karadeniz Yildirim, arXiv:2104 .13074.

[55] P.-P. Shi, F. Huang, and W.-L. Wang, Phys. Rev. D 103, 094038 (2021).

[56] S. Brodsky, D. Hwang, and R. Lebed, Phys. Rev. Lett. 113, 112001 (2014).

[57] R. Lebed, Phys. Lett. B 749, 454 (2015).

[58] R. Lebed, Phys. Rev. D 96, 116003 (2017).

[59] J. Giron, R. Lebed, and C. Peterson, J. High Energy Phys. 05 (2019) 061.

[60] J. Giron, R. Lebed, and C. Peterson, J. High Energy Phys. 01 (2020) 124.

[61] J. Giron and R. Lebed, Phys. Rev. D 101, 074032 (2020).

[62] J. Giron and R. Lebed, Phys. Rev. D 102, 014036 (2020).

[63] J. Giron and R. Lebed, Phys. Rev. D 102, 074003 (2020).

[64] J. Gens, J. Giron, and R. Lebed, Phys. Rev. D 103, 094024 (2021).

[65] R. Lebed and A. Polosa, Phys. Rev. D 93, 094024 (2016).

[66] K. Juge, J. Kuti, and C. Morningstar, Phys. Rev. Lett. 90, 161601 (2003). 
[67] http://www.andrew.cmu.edu/user/cmorning/static_potentials/ SU3_4D/greet.html.

[68] S. Capitani, O. Philipsen, C. Reisinger, C. Riehl, and M. Wagner, Phys. Rev. D 99, 034502 (2019).

[69] S. Prelovsek, S. Collins, D. Mohler, M. Padmanath, and S. Piemonte, J. High Energy Phys. 06 (2021) 035.

[70] R. Aaij et al. (LHCb Collaboration), Phys. Rev. Lett. 118, 022003 (2017).

[71] T. Skwarnicki (private communication).

[72] R. Aaij et al. (LHCb Collaboration), Phys. Rev. Lett. 122, 222001 (2019).
[73] R. Aaij et al. (LHCb Collaboration), Phys. Rev. Lett. 115, 072001 (2015).

[74] J.-J. Wu, R. Molina, E. Oset, and B.-S. Zou, Phys. Rev. Lett. 105, 232001 (2010).

[75] W.-L. Wang, F. Huang, Z.-Y. Zhang, and B.-S. Zou, Phys. Rev. C 84, 015203 (2011).

[76] Z.-C. Yang, Z.-F. Sun, J. He, X. Liu, and S.-L. Zhu, Chin. Phys. C 36, 6 (2012).

[77] M. Karliner and J. Rosner, Phys. Rev. Lett. 115, 122001 (2015). 